\title{
résistance au cisaillement dynamique
}

\author{
dynamic shear strength
}

\author{
Note de Pierre LONDE \\ Ingénieur-Conseil, Pierre Londe \& Associés* \\ Président Honoraire de la Commission Internationale des Grands Barrages
}

\section{Résumé}

Des essais de cisaillement direct très rapides, faits sous la direction du Professeur Skempton, ont montré que la résistance de surfaces précisaillées croissait en général avec la vitesse de cisaillement. Mais certains silts posent un sérieux problème dynamique en manifestant une chute en dessous de la résistance résiduelle. Aucune explication n'a encore été avancée.

\section{Abstract}

Fast direct shear tests, carried out under the guidance of Professeur Skempton, have shown that in general the strength of pre-sheared surfaces increased substantially for fast shear. However some silt-stone displayed a drop below the residual strength, raising a serious problem for dynamic design. No explanation has been proposed thus far.

\footnotetext{
- Tour Horizon - 92806 Puteaux Cedex.
} 
1. Dans le cadre de l'étude du barrage de Kalabagh, situé au Pakistan dans une région fortement sismique $(\mathrm{M}=7, \mathrm{a}=0,4 \mathrm{~g})$, le professeur SKEMPTON a été invité à donner son avis sur la résistance des plans de cisaillement d'origine tectonique qui règnent sur de grandes surfaces dans la fondation. Celle-ci est constituée de bancs alternés de grès (sandstone) et de marnes (claystone et siltstone). Ce sont les marnes qui comprennent des zones précisaillées subhorizontales de grande étendue.

Le Professeur SKEMPTON a fait l'étude sur des échantillons de roches du site (siltstone et claystone), en utilisant la machine de cisaillement par torsion annulaire de BISHOP. Les échantillons ont $19 \mathrm{~mm}$ d'épaisseur, $100 \mathrm{~mm}$ de diamètre intérieur et $150 \mathrm{~mm}$ de diamètre extérieur. Ils sont consolidés sous $900 \mathrm{kPa}$ avant d'être cisaillés sous des contraintes normales inférieures $(500$ ou $200 \mathrm{kPa})$. Les vitesses de cisaillement ont été de $0,01 \mathrm{~mm} / \mathrm{min}$ (essai lent 'dit "statique »), $10 \mathrm{~mm} / \mathrm{min}, 100 \mathrm{~mm} / \mathrm{min}, 400 \mathrm{~mm} / \mathrm{min}$ et $700 \mathrm{~mm} / \mathrm{min}$ (limite de la machine).

Les résultats de SKEMPTON sont donnés ci-dessous, d'après un rapport interne rédigé par lui-même. Voir aussi le Xle Congrès International de Mécanique des Sols et des Travaux de Fondation. L. LEMOS A.W. SKEMPTON, P.R. VAUGHAN «Earthquake loading of shear surfaces in slopes $» .7 / B / 4$.
2. Dans l'état actuel des connaissances on ne peut donner que des explications hypothétiques aux comportements observés dans les essais très rapides.

2.1. Pour les faibles pourcentages d'argile des siltstones (fig. 1), le matériau acquiert d'abord une résistance supplémentaire, qui peut être associée à la dilatance et à l'apparition des pressions interstitielles négatives associées, en tout cas dans le plan de cisaillement. Mais ces pressions interstitielles peuvent se dissiper rapidement dans l'échantillon, au cours du déplacement, et ce faisant elles conduisent à une perte progressive de résistance. Il est important de remarquer que la résistance en essais très rapides ne tombe jamais au-dessous de la résistance statique (essai lent à $0,01 \mathrm{~mm} / \mathrm{min}$ ) et qu'il n'existe pas de pic lorsqu'on fait ensuite un essai lent sur le même échantillon.

2.2. Pour les forts pourcentages d'argile des claystones $(>40 \%)$ le gain de résistance en essai très rapide (fig. 2) peut venir principalement d'un remaniement de la structure initiale, qui était constituée de particules d'argile parallèles à la direction du cisaillement : on peut proposer l'analogie d'un cisaillement « turbulent », différent du cisaillement «par glissement ». Il se peut aussí que des pressions interstitielles négatives jouent leur rôle, mais l'hypothèse du désordre de structure est

\section{$3 \%<0.002 \mathrm{~mm}$}

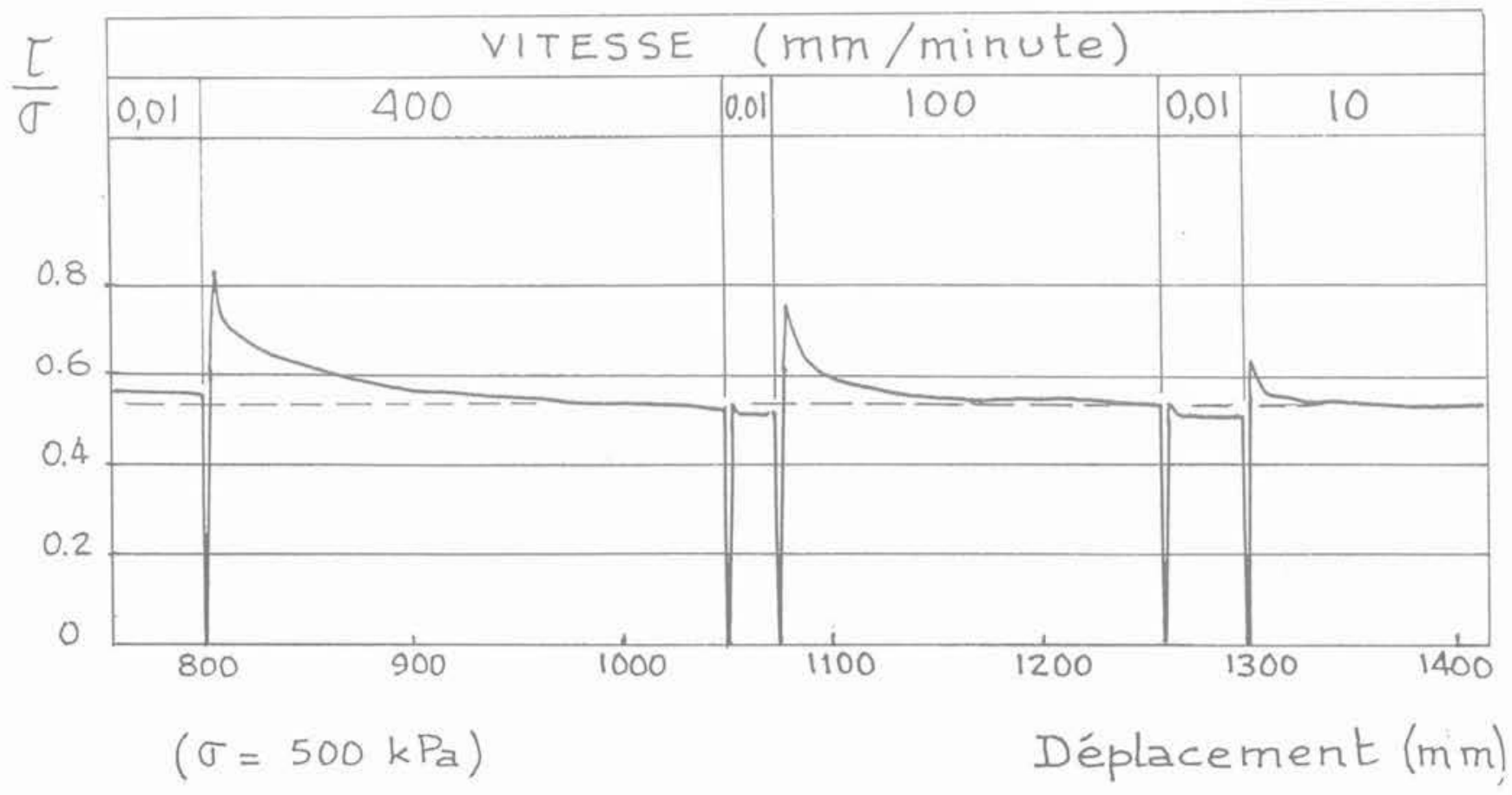

Fig. 1. 


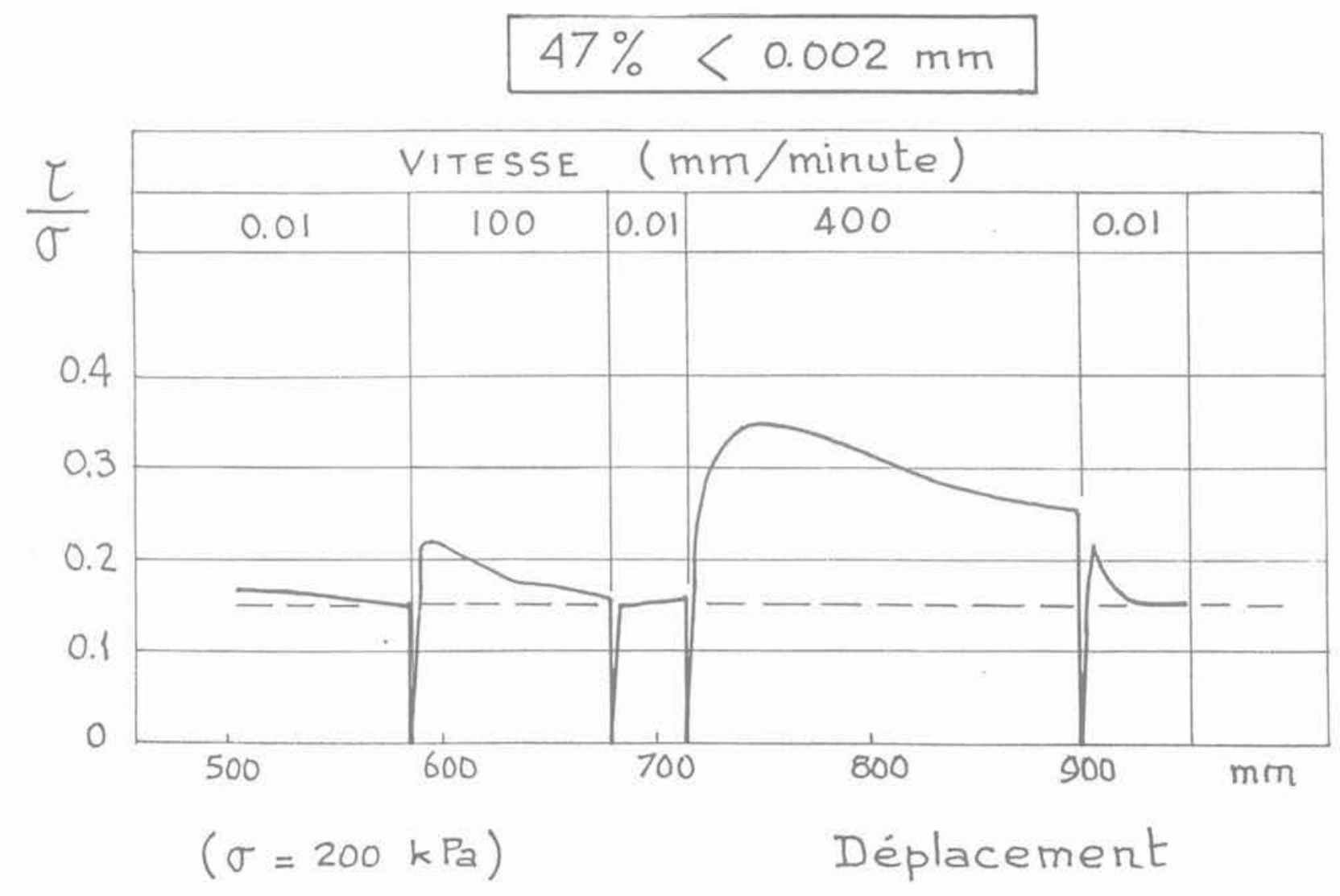

Fig. 2.

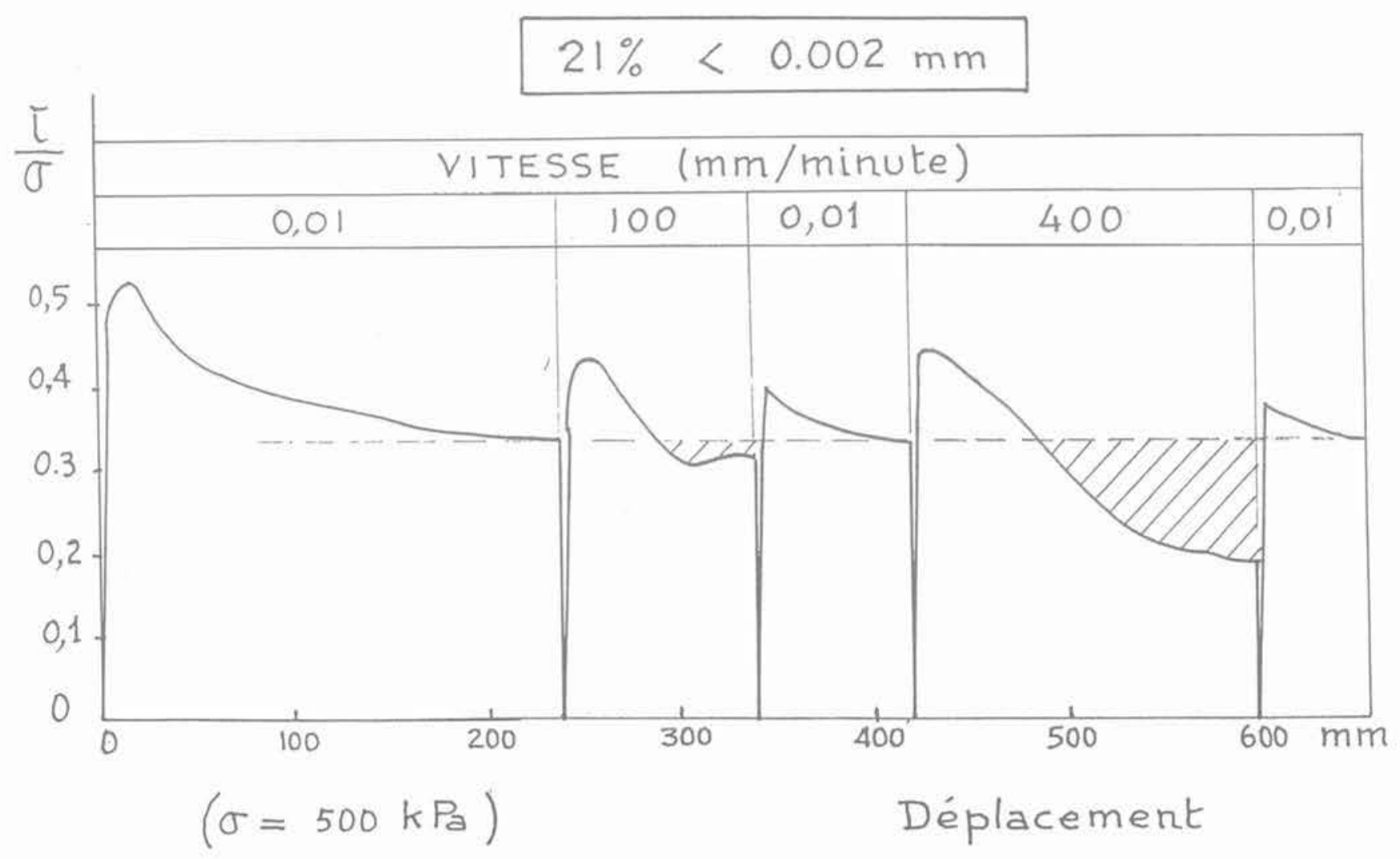

Fig. 3. 


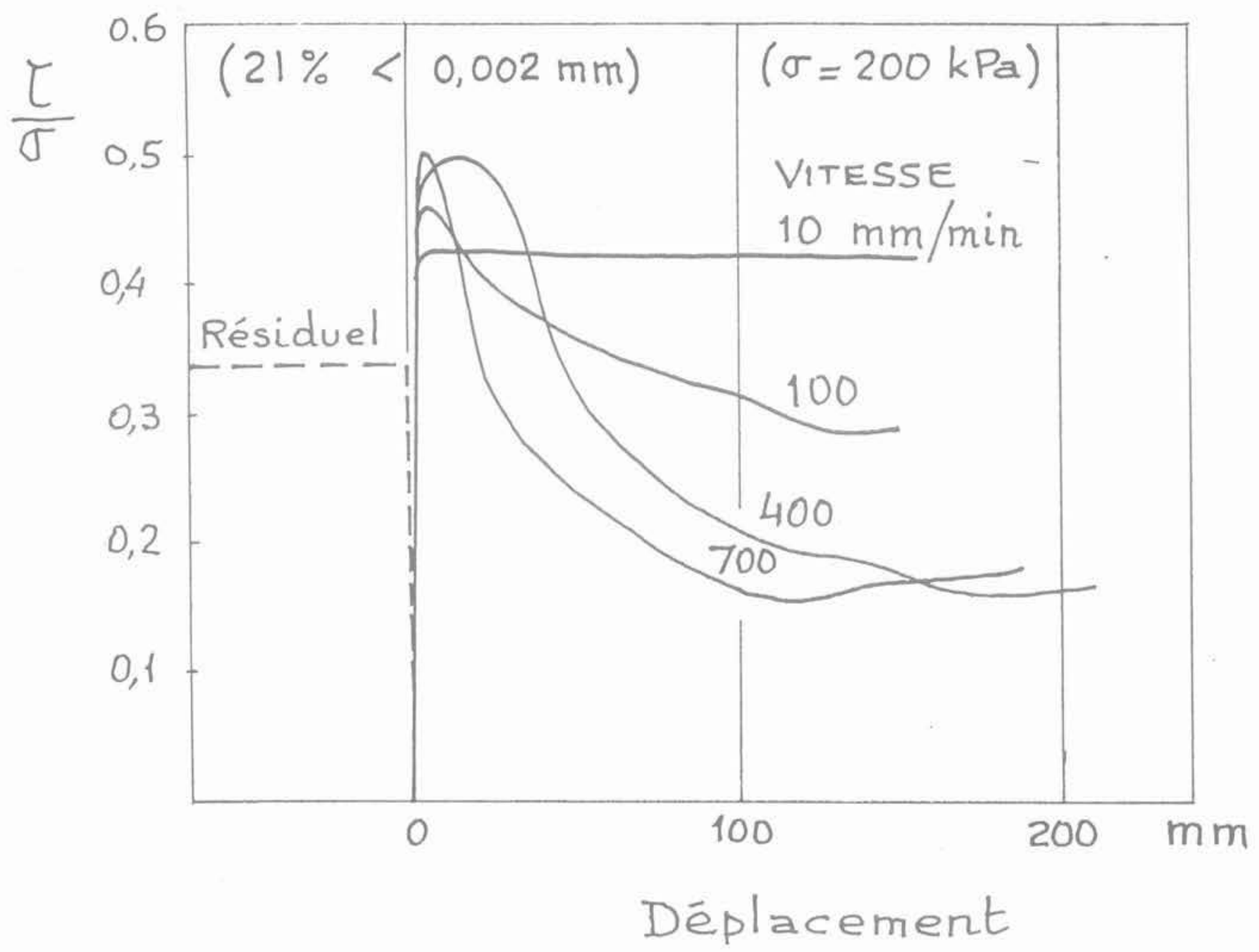

Fig. 4.

étayée par le pic marqué qu'on observe lorsqu'on applique à nouveau un cisaillement lent, un grand déplacement étant alors nécessaire pour retrouver la valeur statique qui correspond à la parfaite orientation des particules.

2.3. On peut s'attendre à ce qu'un échantillon de siltstone argileux se situe en transition entre le comportement du siltstone pur et celui du claystone. C'est-à-dire que les matériaux de cette zone de transition ne sont ni des silts avec un peu d'argile, ni des argiles avec des grains de silt sans contact entre eux. Mais alors pourquoi les matériaux de transition ont-ils le comportement qu'on observe (fig. 3) : un gain "normal» de résistance en essai rapide, suivi d'une chute «anor. male». Cet effet est accentué par une augmentation de la vitesse (fig. 4). Aucune explication n'a été trouvée à ce jour.

Quoi qu'il en soit, et bien que les explications soient ou hypothétiques ou manquantes, l'effet de la vitesse de cisaillement sur la résistance de surfaces préexistantes a été mesuré et cet effet répond à un schéma régulier : un accroissement de résistance, avec un pic marqué, suivi d'une chute progressive ramenant la résistance à la valeur résiduelle statique, ou même très en dessous pour les matériaux moyennement argileux.

Les paramètres caractéristiques $\varnothing$, (essai lent) et $\varnothing_{\max }$ et $\varnothing_{\min }$ (essai très rapide, $400 \mathrm{~mm} / \mathrm{min}$ ), pour $\sigma=400 \mathrm{kPa}$, sont donnés en fonction du pourcentage d'argile dans la figure 5 . Cette figure résume commodément les principaux résultats de l'étude. La courbe $\varnothing$, confirme les résultats des essais de císaillement alternés.

3. Aujourd'hui, et pour les applications pratiques, on peut tirer les conclusions suivantes :

3.1. Tous les échantillons cisaillés à grande vitesse donnent un accroissement de résistance (par rapport à la valeur "statique»), et ce gain est obtenu pour de petits déplacements additionnels.

3.2. Aux grands déplacements tous les échantillons cisaillés à grande vitesse manifestent, mais à des degrés divers, un comportement "radoucissant ". Dans les argiles cet effet est minimal. Dans les silts argileux, et 


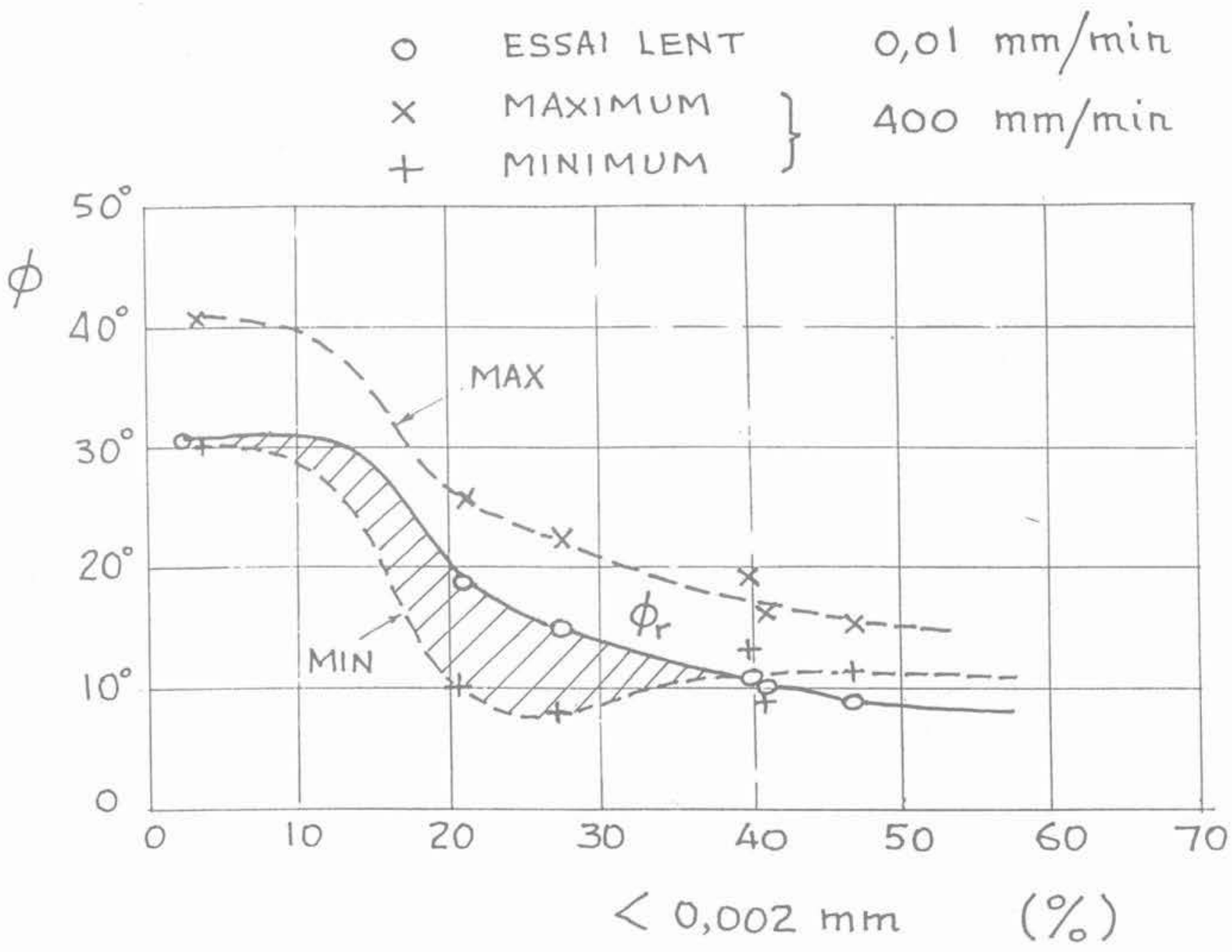

Fig, 5 .

même dans les silts peu argileux, le radoucissement est suffisamment marqué pour créer un problème à l'ingénieur (fig. 6). Il semble aggravé par l'augmentation de la vitesse de cisaillement.

3.3. Lorsqu'on reprend l'essai à vitesse lente, tous les échantillons cisaillés retrouvent (ou même dépassent) leur résistance résiduelle statique.

4. Cette étude montre que pour pouvoir profiter du gain de résistance que génèrent les sollicitations très rapides (séisme), il faut s'assurer que cette résistance est suffisante pour s'opposer aux grands déplacements. Sinon, le radoucissement fait perdre tout le bénéfice et peut même résulter en une résistance beaucoup plus faible qu'avant la sollicitation dynamique.

Peut-être faut-il voir là l'explication des grands glisse- ments provoqués par certains séismes et mal expliqués à ce jour.

Quoi qu'il en soit, on est encore loin de pouvoir déterminer quantitativement cet effet. Le travail de SKEMP. TON n'est qu'un début et il mérite d'être poursuivi. Il y a là un beau défi pour les laboratoires et les constructeurs de machines d'essais : faire un appareil de cisaillement én torsion qui applique un déplacement de $5000 \mathrm{~mm}$ par minute, soir environ $10 \mathrm{~cm}$ par seconde, et cela pendant plusieurs secondes.

L'enjeu est considérable. Car s'il n'est pas trop grave de négliger un surcroît de résistance, faute de pouvoir le quantifier, il est beaucoup plus grave d'ignorer l'effondrement de résistance qui lui succède. Cet effondrement a été observé au laboratoire, pour des silts moyennement argileux. La menace est grave et l'étude du phénomène doit passer avant le raffinement des modèles mathématiques. 


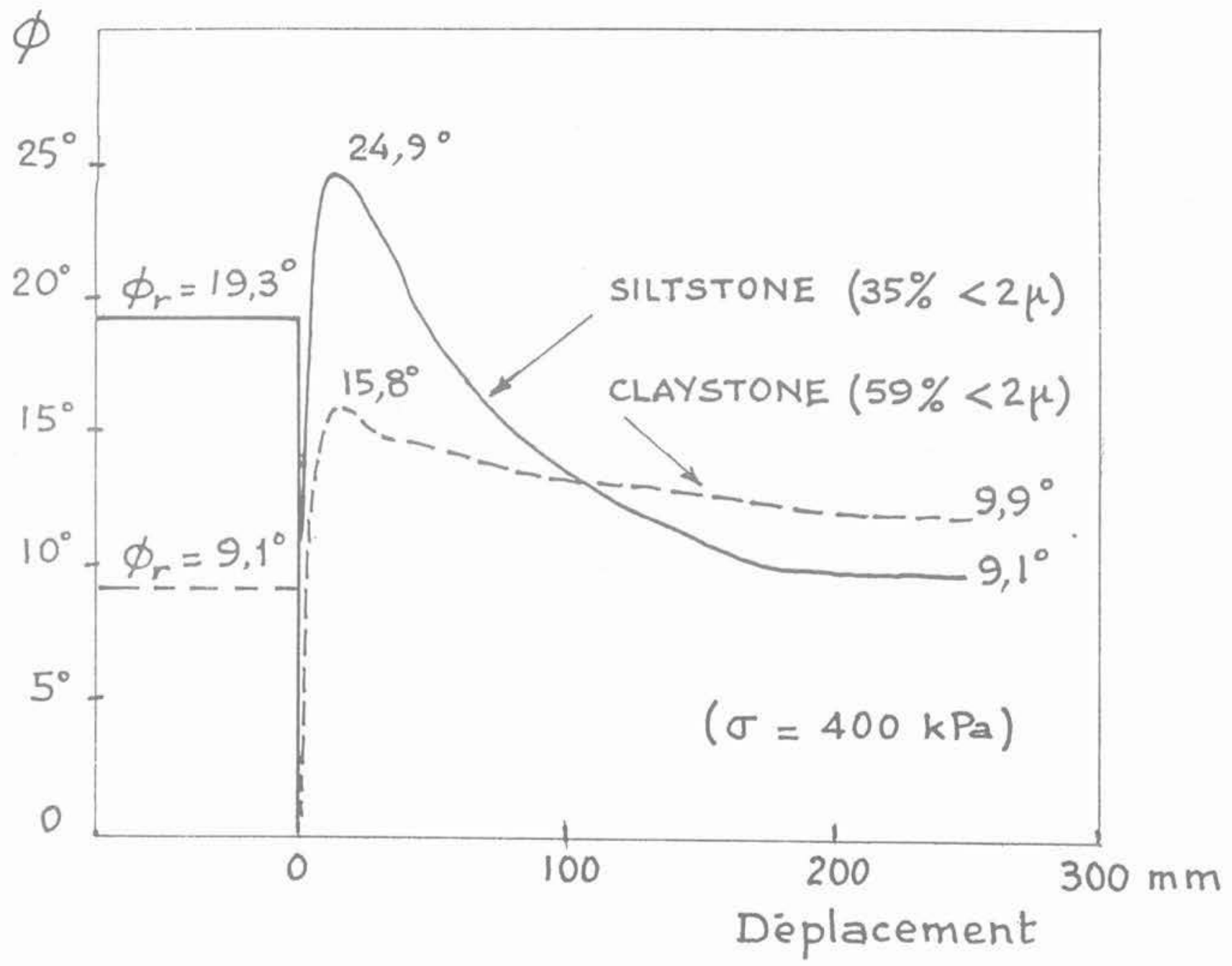

VItesse: $400 \mathrm{~mm} /$ minute

Fig. 6. 